\title{
Le lait, une matière d'avenir pour la cosmétique
}

\author{
J Cotte \\ Institut de pharmacie industrielle de Lyon, université Claude Bernard, \\ 8, avenue Rockefeller, 69008 Lyon, France
}

Résumé - La cosmétologie est maintenant devenue une discipline scientifique à part entière. Elle s'appuie sur une démarche et des expérimentations rigoureuses ayant pour objet de démontrer l'efficacité des produits testés dans 2 grands domaines : la protection active de la peau ou la relance par stimulation du métabolisme cutané.

Les composants du lait, sécrétion naturelle utilisée en cosmétologie depuis l'antiquité, répondent dans de nombreux domaines aux besoins de la cosmétologie. Les protéines du lait sont à même de constituer des principes actifs hydratants; elles peuvent également se substituer aux tensio-actifs de synthèse dans l'élaboration des shampooings. De plus, celles comportant une fraction glycosylée pourraient être largement utilisées dans tous les produits de lutte contre le vieillissement cutané. Enfin, la lactoferrine, du fait de sa capacité à chélater le fer, serait à même de prévenir le développement de radicaux libres découlant de l'exposition solaire.

S'ils peuvent être séparés et purifiés à des coûts acceptables, les composants de la membrane des globules gras du lait pourraient trouver également de larges débouchés en cosmétologie, que ce soit en tant que principes actifs ou pour la réalisation de systèmes nanovésiculaires (liposomes).

cosmétologie / protéine du lait / phospholipide / céramide / lactoferrine / lactoperoxydase / propriété fonctionnelle

Summary - Future of milk components in cosmetology. Cosmetology has now become a fully scientific discipline. It uses rigorous experimentations and methods of reflection for demonstrating the efficiency of tested products in 2 main areas: active skin protection and stimulation of cutaneous metabolism. Because of their natural origin, milk components correspond in many fields to the needs of cosmetology. Milk proteins could be active substances for hydration; they could also substitute synthetic surfactants and so lead to non agressive shampoos. Moreover, those with a glycosylated part could be largely used in all products developed to fight against skin ageing. Lactoferrin, which has a known high iron chelating property could prevent free radicals production by the skin after long periods of exposure to the sun.

If they can be separated and purified at economically acceptable costs, fat globule membrane components, such as phospholipids or ceramids could be widely used in cosmetology, either as active drugs or for obtaining nanovesicular systems as liposomes.

cosmetology / milk protein / phospholipid / ceramid / lactoferrin / lactoperoxidase / functional property 
Le thème qui m'a été confié se distingue des autres exposés en ce sens qu'il aborde un domaine assez inattendu dans une réunion comme la vôtre.

Je ne ferai pas référence aux derniers travaux scientifiques concernant cette matière première. J'en suis bien incapable devant les spécialistes que vous êtes, mais je souhaiterais attirer votre attention sur les possibilités d'application de certains constituants ou de certaines fractions du lait, qui ouvrent en cosmétologie des perspectives d'autant plus intéressantes qu'elles s'inscrivent dans une actualité très favorable.

Dès maintenant, on peut véritablement se poser la question d'une diversification cosmétique des activités traditionnelles laitières, au moment où d'autres secteurs agro-alimentaires s'engagent avec certains moyens dans cette voie.

Non seulement on vise un enrichissement de ces activités, mais aussi on peut valoriser des sous-produits dont l'intérêt commercial est aujourd'hui pratiquement nul.

Avant de vous faire part de mes réflexions sur les possibilités cosmétiques des constituants les plus significatifs du lait (ma liste sera loin d'être exhaustive, je me contenterai de quelques exemples), je souhaiterais dans une première partie vous entretenir des spécificités de la cosmétologie. Cette discipline ou cette activité présentent en effet des caractéristiques tout à fait particulières qu'il est nécessaire de bien connaître pour appréhender ce marché.

\section{SPÉCIFICITÉS DE LA COSMÉTOLOGIE}

J'aborderai successivement 3 aspects :

- conception actuelle de la cosmétologie;

- le concept de biocompatibilité;
- positionnement cosmétique des matières premières.

\section{Conception actuelle de la cosmétologie}

Vous devez être persuadés que la cosmétologie est maintenant devenue une science s'appuyant sur des faits précis d'ordre biologique et physicochimique. Je n'en veux pour preuve que la reconnaissance universitaire de cette discipline dont nous avons été l'initiateur il y a plus de 30 ans.

En effet, le produit cosmétique n'est plus ce produit qui devait tout à l'artificiel ou au faux-semblant dans le but de donner l'illusion d'une réalité ou plus, de cacher cette réalité. Sans sous-estimer cette cosmétologie d'apparat, parfumage et maquillage, le produit cosmétique au sens où nous l'entendons est devenu une préparation toujours testée au point de vue de sa tolérance et douée de propriétés hygiéniques, capable de rendre ou de conserver à la peau son équilibre physico-chimique sans affecter les fonctions de l'organisme, c'est-à-dire sans intention d'effet pharmacologique.

Le produit cosmétique n'est pas un médicament, en ce sens que son action ne ressort pas de la pathologie, mais qu'il agit essentiellement en maintenant ou en rétablissant un bon état physiologique.

On peut shématiquement distinguer 2 types d'action cosmétique :

- protection active palliant les effets nocifs de facteurs d'origines diverses provoquant ou accentuant sa détérioration (vieillissement, agents climatiques, médicaments);

- relance par stimulation du métabolisme cutané provisoirement assoupi ou agressé.

Dans le premier cas, le produit cosmétique va permettre de prolonger le mieux et le plus longtemps possible le bon état de 
la peau en rétablissant les conditions d'un bon équilibre physiologique.

Par protection active on entend non pas une mise sous cloche du tégument mais une protection tenant compte des échanges cutanés par des produits faisant appel à des constituants rigoureusement sélectionnés pour leur affinité la plus stricte avec le gel épidermique.

La cosmétologie acquiert ainsi sa véritable dimension, prévention dans le domaine de l'hygiène quotidienne et, pour ne nous en tenir qu'au vieillissement cutané, cible de nombreux produits cosmétiques; il ne s'agit pas de retrouver par un coup de baguette magique à 80 ans la peau de ses 20 ans, mais de conserver le plus longtemps possible la peau de ses 20 ans.

Dans le deuxième cas, la cosmétologie ne limite pas son rôle au seul effet protecteur actif. Elle peut également permettre une réactivation des moyens naturels qui assurent le métabolisme cutané, dans la mesure où ils ont subi l'atteinte d'agressions diverses dont celles d'origine médicamenteuse qui en ont diminué le bon déroulement.

Cette conception réaliste, raisonnable et scientifique de la cosmétologie explique l'évolution santé de la cosmétologie.

Le produit cosmétique trouve en effet sa place dans le domaine de la santé prise dans son sens le plus large : n'oublions pas la définition qu'en donne I'OMS "état de complet bien-être et non pas seulement absence de maladie".

On peut donc parler d'une véritable efficacité cosmétique qui ne résulte pas de seules considérations théoriques plus ou moins fantaisistes. Cette efficacité est mise en évidence grâce à des tests objectifs et non plus par les seuls tests d'usage ou cliniques. Ces tests sont basés sur des méthodologies rigoureuses qui ont permis de faire progresser nos connaissances de physiologie et de biomécanique cutanée.
L'appréciation des effets résulte de l'exploitation statistique de nombreuses données. On sait maintenant mesurer de façon scientifique les principales propriétés de la peau : hydratation, souplesse, élasticité, coloration, état de surface, profilométrie, etc.

Les formulateurs exigent de leurs fournisseurs des dossiers importants de tolérance et d'activité, et chaque produit terminé est testé avant sa mise sur le marché, en ce qui concerne ses propriétés les plus significatives. Ces travaux sous-tendent le plus souvent l'argumentaire marketing et sont effectués avec la plus grande rigueur scientifique.

II y a donc là une situation qu'il convient de ne pas négliger. La cosmétologie a acquis ses lettres de noblesse en tant que discipline scientifique et de ce fait, peut être prise en considération pour la qualité de sa démarche dans la conception des produits qui relèvent de son application. Des dermatologues ne s'y sont pas trompés en intégrant les produits cosmétiques dans leur pratique médicale.

La formulation de ces produits s'appuie en effet sur des connaissances maintenant bien établies de la physiologie cutanée. Elle fait appel à des constituants, actifs, obéissant à des spécifications physicochimiques rigoureuses dont l'efficacité doit être mise en évidence grâce à une méthodologie dont l'objectivité est indiscutable.

De plus, un produit cosmétique est un tout, en ce sens qu'il n'est pas constitué, comme son analogue médicamenteux du seul couple «principe actif-excipient», ce dernier n'ayant pour rôle que d'être au seul service du principe actif en optimisant son action.

Dans un produit cosmétique, tous les constituants contribuent à l'activité finale, y compris même la texture qui a toujours une part active dans la protection et le ré- 
tablissement des propriétés normales de la peau.

II existe ainsi une globalité de la formulation cosmétique qui explique que cette dernière est toujours extrêmement riche, non comparable à celle d'une pommade ou d'un onguent chez lesquels la base est là pour permettre seulement l'application du ou des principes actifs.

\section{Concept de biocompatibilité}

Cette notion devient maintenant primordiale dans le choix des différents composants d'une formule cosmétique.

Par biocompatibilité il ne faut pas seulement entendre tolérance parfaite mais plutôt affinité étroite avec les constituants cutanés. Cette affinité est due à l'origine biologique des différents composés utilisés, et on cherche même à donner la préférence à ceux présentant une ressemblance ou une analogie avec les constituants cutanés. On parle de substances épidermomimétiques. D'où la vogue des extraits biologiques ou de molécules définies telles le collagène, l'élastine etc, mais il existe dans ce domaine un handicap sérieux depuis les prises de position agressives des défenseurs des animaux. Dans ce contexte, I'origine animale des extraits biologiques est souvent dénoncée violemment par des groupes de pression organisés.

II existe une réelle contradiction entre de telles manifestations et l'exigence du "naturel" revendiqué par les mêmes associations au nom du respect de l'environnement. Les utilisateurs doivent tenir compte de ce courant qui n'est plus une mode, d'autant que les "extraits naturels" bénéficient d'un agrément réglementaire quasi automatique, ce qui n'est pas le cas des nouvelles molécules synthétiques. On se tourne alors vers le réservoir végétal mais l'efficacité cosmétique des fractions extraites reste encore limitée.

II existe donc pour le lait, sécrétion physiologique naturelle, une opportunité exceptionnelle de répondre aux demandes d'une industrie condamnée à un renouvellement constant de ses formules et à une adaptation aux exigences du marché.

\section{Positionnement cosmétique des matières premières}

Nous avons dit que la formulation cosmétique fait intervenir de nombreux composants. Se pose alors le problème de leur compatibilité, ce qui explique la tendance qui se développe actuellement de proposer des matières premières "pré-formulées", c'est-à-dire profilées pour l'usage auquel elles sont destinées. Une nouvelle situation est ainsi créée, de transfert vers l'amont d'une certaine valeur ajoutée. Les conséquences de ce transfert sont importantes sur l'évolution de la notion même de matière première. Le marché est demandeur - et plus spécifiquement en cosmétologie - non pas de produits chimiques banalisés présentés pour tous usages, mais de la réponse à un besoin technicobiologique précis.

II apparaît ainsi un créneau commercial nouveau tout à fait prometteur, qui consiste à partir d'une matière première déterminée à en décliner plusieurs types, chacun étant destiné à une application particulière. On peut même imaginer un label spécial garantissant une qualité de constituant en prise directe avec le domaine d'application pour lequel ils ont été conçus.

Tout le monde y trouve son compte :

- le fabricant qui fidélise son client et développe son profit par la valeur ajoutée;

- l'utilisateur qui est intéressé par l'amélioration de la faisabilité de son produit et la 
solution rapide au problème posé par le marketing.

\section{QUELQUES EXEMPLES \\ D'APPLICATIONS COSMÉTIQUES POSSIBLES DE DÉRIVÉS DU LAIT}

Nous n'avons pas l'intention de faire l'inventaire de tous les constituants du laits ni de les étudier sous l'angle de leurs possibles applications cosmétiques.

Nous nous limiterons à quelques produits qui ne sont peut-être pas les plus significatifs, mais qui sont déjà proposés aux formulateurs cosmétiques ou qui sont susceptibles de développements nouveaux.

Ces produits peuvent être utilisés, soit comme adjuvants technologiques, soit comme actifs conférant aux formules dans lesquelles ils sont incorporés, des propriétés cosmétiques spécifiques.

Leur intérêt est lié à leur origine naturelle. Ils peuvent venir se substituer à d'autres composants doués des mêmes propriétés, mais ne bénéficiant pas du même label. En outre, la meilleure connaissance de leurs possibilités peut conduire à la définition de nouveaux concepts d'efficacité cosmétique.

Nous prendrons quelques exemples dans les grandes familles chimiques et nous terminerons par quelques réflexions sur certaines voies de recherches possibles.

\section{Les composés azotés}

\section{Propriétés générales des protéines applicables en cosmétologie}

Parmi les propriétés générales des protéines applicables en cosmétologie nous retiendrons :

\section{Leur pouvoir hydratant}

Les protéines ont une forte capacité d'absorption et de rétention d'eau. Cette propriété peut être mise à profit comme adjuvant technologique dans de nombreuses préparations émulsionnées, crèmes ou laits, comme stabilisant de la formulation. Cette mouillabilité leur confère un effet de substantivité, c'est-à-dire de meilleure adhésivité avec la structure cutanée. Mais surtout, elles peuvent et doivent constituer des actifs hydratants parfaitement physiologiques. En effet, les préparations cosmétiques hydratantes sont, pour la plupart, des produits antidéshydratants dans la mesure où leur formule fait appel à des substances retenant fortement l'eau et assurant ainsi à la surface de la peau un degré d'hydratation maximale. Ce microclimat humide permanent stoppe la perte en eau du stratum corneum réalisant ainsi une véritable protection active telle que nous l'avons définie. La peau retrouve alors un état normal et un aspect esthétique "rajeuni", les ridules étant estompées.

\section{L'aptitude à la gélification}

C'est une propriété fonctionnelle de la plupart des protéines dont peuvent bénéficier de nombreuses textures cosmétiques. Les protéines du lactosérum manifestent de bonnes propriétés gélifiantes très proches de celles du blanc d'œuf. Elles peuvent être utilisées comme adjuvant de liaison et de viscosité.

\section{Leurs propriétés émulsifiantes}

Les agents de surface sont des constituants prédominants des cosmétiques. On est tenté de voir une similitude entre les tensio-actifs synthétiques à longue chaîne, comprenant des radicaux alkyls, aminés et carboxyliques et les protéines. Les protéines sont adsorbées à l'interface, entre les gouttelettes d'huile dispersées et la phase aqueuse continue, mais ce sont gé- 
néralement de médiocres stabilisants d'émulsions eau dans huile du fait de la nature principalement hydrophile de la plupart des protéines. Dans le même ordre d'idées, on peut comparer les tensio-actifs aux condensats de protéines et d'acides gras. L'effet mouillant de ces produits est faible, mais leur pouvoir émulsifiant et dispersant est bon. Leur tolérance est parfaite et leur emploi est fréquent dans les shampooings (shampooings aux protéines).

\section{Leurs propriétés moussantes}

Certaines protéines possèdent de bonnes propriétés moussantes et en particulier les protéines du lactosérum et le caséinate de sodium.

Depuis longtemps le consommateur est habitué à associer le moussage abondant à un bon pouvoir nettoyant et un détergent non moussant apparaît comme insatisfaisant.

La vogue des bains moussants est maintenant généralisée mais on connaît l'effet détergent agressif des tensio-actifs synthétiques. On pourrait donc envisager l'adjonction à ces bains moussants de poudre de lait spray ou de caséinate de sodium. Leur action douce, leur origine naturelle, la légende beauté qui s'attache depuis Poppée et Cléopâtre aux bains de lait sont autant de raisons pour orienter les formulateurs vers cette nouvelle classe de constituants.

\section{Protéines du lactosérum}

Elles sont principalement constituées de $\beta$ lactoglobuline et d' $\alpha$-lactalbumine. On a préconisé leur utilisation en solution relativement concentrée pour leur effet tenseur filmogène, effaçant temporairement les rides : action coup d'éclat.

On les utilise également comme agents hydratants soit purs, soit sous forme de lactosérum enrichi en fractions protéiques.

\section{Caséine}

Outre son utilisation pour ses propriétés émulsifiantes et moussantes, la caséine est une matière première pouvant donner naissance à des constituants du plus grand intérêt cosmétique. Nous citerons plus particulièrement :

\section{L'hydrolysat de caséine}

II contient les acides aminés en proportions physiologiques et cette composition est relativement proche de celle que l'on trouve dans le NMF (natural moisturising factor).

Le NMF, comme son nom l'indique est le facteur naturel d'humidification maintenant l'hydradation normale de la peau : ce facteur fixe à son optimum la teneur en eau de la couche cornée.

Il provient de la dégradation d'une protéine épidermique, la filaggrine. Cette dégradation a lieu lorsque l'humidité relative est basse. Elle conduit à un mélange de certains constituants qui sont à l'origine du NMF. Parmi ces composants, on trouve des acides aminés libres qui jouent un rôle dans la régulation de l'hydratation cutanée. L'hydrolysat de caséine représente un contretype des acides aminés du NMF et à ce titre renforce l'hydratation épidermique.

Ces complexes d'acides aminés jouent également un rôle important par leur pouvoir tampon dans le maintien du $\mathrm{pH}$ cutané, épongeant tout excès d'acide ou d'alcali et protégeant ainsi la peau de l'action 
agressive de facteurs environnementaux d'origine diverse.

\section{Glycoprotéines du lait}

Sous cette dénomination, on désigne la fraction glycoprotéinique soluble obtenue après action de la présure sur la caséine $\kappa$. II s'agit de phospho-glyco-caséinomacropeptides comprenant une partie phosphopeptidique fixe et une partie glucidique variable. Ce produit obtenu est purifié par ultrafiltration (brevet UCALM 1976).

Les glycoprotéines sont des constituants principaux de la substance fondamentale du tissu conjonctif. Associées aux mucopolysaccharides elles jouent un rôle important quoiqu'encore mal connu dans le système de maintien de l'intégrité des fibres de la peau.

La principale propriété cosmétique des glycoprotéines est leur pouvoir hydratant, essentiellement dû à la partie glucidique de la molécule. En effet, du fait de l'affinité des glucides et tout particulièrement de l'acide sialique pour l'eau, et des propriétés intrinsèques des protéines, on ne peut s'attendre pour les glycoprotéines qu'à des résultats remarquables en matière d'hydratation cutanée. Ces effets ont été vérifiés par des mesures d'impédance in vivo sur la peau humaine.

Par ailleurs, il convient de noter le rôle des glycoprotéines dans l'augmentation du pouvoir hydratant de certaines préparations contenant des MPS (mucopolysaccharides et plus particulièrement l'acide hyaluronique. En effet, les glycoprotéines forment naturellement avec les MPS des associations moléculaires faisant intervenir un grand nombre de molécules d'eau, supérieures au cas oủ ces espèces moléculaires sont prises séparément.

Non seulement, les glycoprotéines peuvent participer indirectement à l'augmentation du pouvoir hydratant de certaines préparations, mais encore, on peut envisager des associations à activités hydratantes optimisées.

Par ailleurs, des travaux récents ont mis en évidence le rôle des glycoprotéines de structure dans le vieillissement de la peau. Cette classe de macro-molécules joue en effet un rôle essentiel dans le maintien de l'organisation de la matrice extracellulaire et dès l'enfance, on observe une diminution progressive de leur biosynthèse.

En dermocosmétologie, afin de maintenir des capacités fonctionnelles du derme, on a déjà réalisé des apports de certaines macromolécules de la matrice extracellulaire, mais les glycoprotéines n'ont pas encore été utilisées à l'exception de la fibronectine.

Les glycoprotéines peuvent être considérées comme un facteur de confort pour les cellules de la peau apportant aux fibroblastes une optimisation des conditions de développement et se comportant comme de véritables bioconnecteurs, renforçant la cohésion du derme.

\section{La lactoferrine}

C'est une glycoprotéine soluble, stable en milieu aqueux, qui a la propriété de fixer 2 ions ferriques par molécule. Elle est considérée comme une protéine ligand inactivatrice du fer quand elle est loin de son niveau de saturation en fer. Dans son état naturel, la teneur en fer de cette protéine ne dépasse pas $25-35 \%$ de son niveau de saturation. Elle montre ainsi une puissante activité complexante pour ce dernier. Or la présence du fer est indispensable à la génération des radicaux libres à partir des peroxydes. Une prévention par blocage de fer apparaît possible à ce niveau. La lactoferrine qui possède la propriété de chelater le fer, le rendra donc indisponible pour la réaction de condensation conduisant à la production du radical hydroxyl. 
On a préconisé l'utilisation de lactoferrine associée à d'autres substances pièges des radicaux libres, dans la prévention du vieillissement cutané.

Les radicaux libres sont produits en grande quantité dans la peau surtout après une exposition au soleil. Ils sont capables d'endommager les lipides cutanés et cette peroxydation lipidique est considérée comme une des causes majeures du vieillissement cutané.

Le succès actuel des préparations antiâge est basé sur leurs propriétés antiradicaux libres et leur efficacité est mise en évidence par des tests précis in vivo et in vitro.

\section{Les lipides}

II ne semble pas que les lipides du lait aient donné lieu jusqu'à maintenant à des applications cosmétologiques précises, et pourtant, le problème lipides et cosmétologie est à l'ordre du jour.

\section{Les céramides}

Nous voudrions plus particulièrement rappeler l'intérêt actuel des céramides intervenant dans la reconstitution du ciment intercellulaire et jouant un rôle fondamental dans la régulation du flux hydrique et le maintien de l'intégrité de la barrière cutanée. Les céramides appartiennent à la classe des sphingolipides (dérivés de la sphingosine et des acides gras). Ils se distinguent des glycosphingolipides où la fonction alcool de la sphingosine est bloquée par un reste sucré.

Ils existent dans le lait sous forme pure et pourraient être avantageusement concentrés si les procédés s'avéraient rentables, l'industrie cosmétique étant fortement demandeuse de ce type de produit.
Leur intérêt a encore augmenté depuis le succès des liposomes où les phospholipides d'origine végétale pourraient connaître déjà, sinon la concurence, du moins la complémentarité des sphingolipides et des céramides.

\section{Les phospholipides}

Cela nous permet de faire la transition avec les phospholipides dont la teneur dans le lait de vache n'est pas négligeable.

Le problème de leur préparation industrielle se pose mais un article récent (Baumy et al, 1990) met en évidence les nouvelles possibilités offertes par les technologies à membranes. II serait théoriquement possible d'extraire $150 \mathrm{~g}$ de phospholipides par 1000 I de lactosérum. Ce pourrait être une matière première précieuse pour la cosmétologie.

\section{Divers}

\section{Le lactosérum}

Parmi les sous produits divers utilisés en cosmétologie, on trouve le lactosérum qui a été préconisé comme agent d'hydratation. II en est de même pour un produit enrichi en protéines et dénommé fraction azotoglucidique.

\section{Le colostrum}

II fait actuellement l'objet d'études dans des directions variées. Il est généralement proposé comme hydratant biologique permettant la rétention et la fixation de l'eau par les cellules épidermiques. Le colostrum est particulièrement riche en lactoferrine.

\section{La lactoperoxydase}

Parmi les constituants existant en faible quantité dans le lait la lactoperoxydase (25 
à $30 \mathrm{mg} / \mathrm{I}$ dans le lait de vache) est préconisée en association avec la lactoferrine dans la normalisation des peaux grasses et le traitement des peaux pré-acnéiques et acnéiques.

Cette action serait due au pouvoir antibactérien du système lactoperoxydase glucose oxydase-thiocyanate de potassium.

Ce système se comporte comme un moteur destiné à produire l'ion hypothiocyanite qui constitue le principe actif possédant l'activité antimicrobienne. Cet ion agit en effet sur les bactéries en oxydant les groupements SH des enzymes responsables du transfert du glucose et des acides aminés provoquant ainsi la dénaturation de ces enzymes et par voie de conséquence, la mort de la bactérie.

Cette action est particulièrement manifeste sur les germes de l'acné et des états pelliculaires, c'est-à-dire les manifestations rattachées à une hyperséborrhée. Aucun dommage ne s'observe sur les cellules, car leur membrane est imperméable à l'hypothiocyanite.

Cependant, il est impossible d'introduire l'ion hypothiocyanite dans un produit cosmétique du fait de son instabilité : il faut donc le produire in situ au niveau de la peau et c'est le rôle du système lactoperoxydasique.

La démonstration de l'activité de ce système a été réalisé par divers tests d'efficacité : diminution du flux séborrhéique, activité comédolytique particulièrement nette à long terme et activité anti-acné (pustules et points-noirs).

La liste que je viens de vous présenter est probablement incomplète et n'obéit à aucune démarche logique dans la recherche de constituants pouvant intervenir dans les produits cosmétiques. II y aurait donc à faire d'abord un état des lieux, c'est-à-dire des besoins cosmétiques et des composants du lait pouvant les satisfaire.

Ensuite, il faudrait ne pas se limiter aux seuls constituants du lait pouvant être utilisés dans des conditions économiques acceptables. Certains d'entre eux, présents en quantité importante pourraient donner lieu à des transformations chimiques aboutissant à des dérivés de grand intérêt cosmétique.

Je vous proposerai 2 exemples:

\section{Les sucro-esters}

Ce sont des substances représentant des molécules intéressantes au point de vue de leur structure chimique. Ce sont en effet des molécules amphiphiles obtenues par estérification du saccharose à l'aide d'acides gras.

Ils peuvent être utilisés comme solubilisants, émulsionnants ou encore épaississants. Ils sont relativement peu connus en Europe et sont peu utilisés en cosmétologie, leur utilisation étant plus courante en alimentation.

Mais comparés à d'autres tensio-actifs non ioniques, les sucro-esters se distinguent par leur parfaite tolérance envers la peau. Ils sont obtenus à partir de produits naturels et peuvent revendiquer une innocuité quasi totale.

Ils s'incrivent donc bien dans le cadre des préoccupations actuelles de la cosmétologie. À notre connaissance, ces esters font actuellement l'objet de recherches en France en vue de leurs applications cosmétiques. Ils retiennent l'attention de groupes agro-alimentaires cherchant à valoriser les excédents de saccharose. On pourrait imaginer que des recherches soient engagées sur une nouvelle classe 
de sucro-esters à base de lactose, la lactosérum pouvant trouver là un débouché intéressant pour sa valorisation.

\section{Les lipo-aminoacides}

L'intérêt des lipoaminoacides comme facteur biologique d'acidification de la peau date des travaux de Morelle en 1967. Cet auteur a montré que l'acidité du revêtement cutané dépend de la teneur en groupement carboxyle des aminoacides dont les fonctions aminées sont acylées par des chaînes lipidiques. Cette acylation aboutit au démasquage de la fonction acide carboxylique de l'acide aminé responsable de l'acidité cutanée d'une part, de sa capacité d'auto-protection contre les microorganismes d'autre part.

Un certain nombre de dérivés ont été synthétisés et sont commercialisés. Les chaînes grasses ont pour origine divers acides $C_{4}, C_{8}, C_{11}, C_{12}, C_{16}$. Les acides aminés peuvent être, soit des acides aminés purs, soit des acides aminés provenant de l'hydrolyse totale des protéines, tels le collagène ou la caséine.

Ils sont utilisés dans la formulation des produits cosmétiques pour leurs propriétés protectrices de la peau et des phanères du fait de leur grande affinité envers la kératine, et, outre leur action sur le maintien et la restauration de l'acidité cutanée et sur le renforcement de l'auto-protection physiologique de la peau, les lipo-aminoacides sont anti-inflammatoires et auraient une activité sur la biosynthèse des protéines.

Par ailleurs, certains lipo-aminoacides ont des propriétés spécifiques et ceux dérivant de l'hydrolysat de caséine exercent une action marquée antiérythémateuse, antiprurigineuse et anti-inflammatoire. Enfin, les sels alcalins des lipoaminoacides sont doués de remarquables propriétés tensio-actives et possèdent un pouvoir moussant élevè. Ce sont des dé- tergents doux, parfaitement tolérés, base des shampooings aux protéines, n'intervenant pas comme le lauryl-sulfate au niveau de la biochimie de la fibre en éliminant les substances protectrices. Ils sont donc particulièrement conseillés pour les shampooings à usage fréquent.

La classe des lipo-aminoacides fait actuellement l'objet de nouvelles recherches orientées vers les propriétés spécifiques de certains dérivés obtenus en faisant varier l'acide acylant, mais surtout le reste azoté.

Le lait, par la diversité de ses constituants azotés devrait avantageusement s'intégrer dans ce programme de recherche en plein développement.

Enfin je voudrais à nouveau attirer votre attention sur les sytèmes nanovésiculaires type liposomes. Je suis profondément persuadé que des liposomes à base de constituants laitiers, céramides en particulier, pourraient avoir une place privilégiée dans les formulations cosmétiques. Ces liposomes d'une autre génération, outre des avantages techniques indiscutables de stabilité susciteraient à l'évidence l'adhésion du consommateur axé sur le retour aux produits vrais et sensibilisés à l'image de marque du lait.

Au terme de cet exposé, j'ai conscience de n'avoir apporté qu'un bilan très parcellaire des possibilités ouvertes par les avancées technologiques et scientifiques de l'industrie laitière. J'ai tenté par quelques exemples choisis pour la plupart dans les dérivés les plus classiques de vous inciter à intégrer dans vos stratégies de valorisation et de diversification, la dimension cosmétique. Cet exposé se réfère davantage à un plaidoyer technico-marketing, qu'à une mise au point scientifique ordonnée.

Il comporte de nombreux oublis, en particulier celui des acides gras insaturés. Et pourtant, nous sommes à l'aube des cons- 
équences importantes qu'auront les recherches des dermatologues sur les dérivés à chaine longue de la famille des eicosanoïdes.

Mais je me situe en bout de chaîne à la partie la plus aval, dans l'attente des résultats de recherches pouvant trouver leur traduction ou leur application en dermocosmétologie.

Je ne voudrais pas terminer sans revenir sur un point important que j'ai évoqué au début de cet exposé, c'est-à-dire, l'adaptation des matières premières à leur application spécifique. Les produits dont nous avons parlé sont en général fragiles, contaminables du fait de leur origine biologique. Leur compatibilité, leur stabilité devront donc faire l'objet d'études rigoureuses avant leur mise sur le marché cosmétique. II en sera de même pour leurs propriétés cosmétiques. Leur présentation pourra donc comporter des ajouts facilitant leur formulation ou optimisant leur activité.

Mais ce secteur d'application cosmétique est bien différent de celui qui est le vôtre, et si j'avais un conseil à donner à ceux qui décideraient de s'engager dans la voie d'un développement cosmétique, ce serait de choisir tout d'abord un partenaire sérieux implanté sur ce marché particulier pour codévelopper les matières premières retenues d'un commun accord. Cette interface me paraît presque obligatoire, au moins dans un premier temps.

Je suis totalement convaincu qu'il y a actuellement une chance à saisir pour le lait dont la richesse et la valeur des constituants, leur caractère naturel et leur parfaite compatibilité biologique sont des atouts exceptionnels pour répondre à la demande pressante d'une industrie soucieuse de fabriquer des produits performants dans le respect des exigences du consommateur.

Et puis, le lait, en tant que nutriment de base indispensable s'inscrit dans le droit fil d'une complémentarité évidente cosmétique/diététique. La synergie entre la voie interne et la voie externe est indispensable au bon fonctionnement des mécanismes physiologiques de la peau, On a pu parler de véritable "pilule de beauté» contribuant au maintien d'un bon état cutané. Cette cosmétologie par voie interne qui pose par ailleurs des problèmes de statut réglementaire, nous semble promise à un plein épanouissement.

J'aimerais emprunter ma conclusion à celle remarquable de Maubois et Léonil (1989) dans un récent article de synthèse sur les peptides du lait :

Bien qu'il soit le liquide alimentaire le mieux connu et le plus transformé, le lait reste encore une source particulierement riche de nouveaux débouchés à haute valeur ajoutée. Les découvertes de la physiologie moderne conduisent à penser que beaucoup de molécules parmi les 2000 qui le composent ont un rôle biologique allant bien au-delà du simple apport de nutriment. II y a là une chance à saisir par tous les acteurs concernés par la filière laitière: producteurs, transformateurs, chercheurs.

J'ajouterai que cette chance est d'autant plus sérieuse que l'industrie cosmétique française connaît une conjoncture exceptionnelle avec une progression moyenne de $15 \% / a n$. Mais tout cela ne sera pas facile, comme le remarquent Maubois et Léonil.

La première question à se poser est de savoir si économiquement la voie des peptides dits naturels est la bonne, si celle des peptides de synthèse ne serait pas plus intéressante; et les auteurs continuent : l'utilisation des peptides de synthèse exige une pureté très élevée en raison des risques de toxicité liés aux solvants et substances chimiques utilisées... ce qui signifie obligatoirement un coût élevé. Une telle pureté n'est pas exigible des peptides na- 
turels si leur préparation a mis en œuvre des processus purement physiques ou physico-chimiques et l'emploi d'enzymes physiologiques.

En peu de mots sont parfaitement situés les avantages incomparables des produits naturels. Au moment où l'utilisation des actifs représentés par des extraits d'origine animale donne lieủ à de vives controverses écologiques, au moment où la mise en évidence objective des propriétés cosmétiques d'extraits végétaux est loin d'être acquise, le lait occupe une place privilégiée qui retiendra à n'en pas douter, de plus en plus l'attention des cosmétologues.

\section{RÉFÉRENCES}

Baumy JJ, Gestin L, Fauquant J, Boyaval E, Maubois JL (1990) Technologie de purification des phospholipides du lactosérum. Process 1047, 29-33

Maubois JL, Léonil J (1989) Peptides du lait à activité biologique. Lait 69, 245-269

Morelle J (1967) Biochimie cutanée et lipoaminoacides de synthèse. Arch Biochim Cosmetol 10, 95-96

Union Coopérative Agricole Laitière de la Manche (UCALM) (1976) Procédé de traitement du lactosérum de fromagerie, notamment en vue de l'extraction de glycoprotéides et d'acide sialique. Brevet FR 2 288-473 